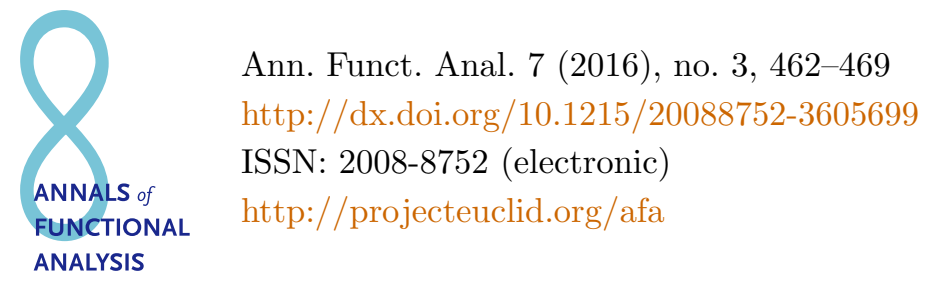

\title{
ABOUT LOCALLY $m$-CONVEX ALGEBRAS WITH DENSE FINITELY GENERATED IDEALS
}

\author{
HUGO ARIZMENDI PEIMBERT ${ }^{1}$ and REYNA MARÍA PÉREZ-TISCAREÑO ${ }^{*}$ \\ Communicated by Z. Lykova
}

\begin{abstract}
It is well known, as a consequence of a theorem of Richard Arens, that a commutative Fréchet locally $m$-convex algebra $E$ with unit does not have dense finitely generated ideals. We shall see that this result can no longer be true if $E$ is not complete and metrizable. We observe that the same is true for the theorem of Arens; that is, this theorem can no longer be true if $E$ is not complete and metrizable. Moreover, several conditions for a unital commutative (not necessarily complete) locally $m$-convex algebra are given, for which all maximal ideals have codimension one.
\end{abstract}

\section{Preliminaries}

Let $E$ be a unital topological algebra over $\mathbb{C}$ (the field of complex numbers) with separately continuous multiplication (in short, a topological algebra).

Let $B$ be a complex commutative unital algebra. We denote by $\mathfrak{M}^{\#}(B)$ the set of all nonzero multiplicative linear functionals on $B$ provided with the weakstar topology $w^{*}$. When $B$ is a topological algebra, $\mathfrak{M}(B)$ denotes the topological subspace of $\mathfrak{M}^{\#}(B)$ consisting of all nonzero multiplicative continuous linear functionals on $B$ provided with the weak-star topology $w^{*}$.

A locally pseudoconvex algebra is a topological algebra with a base of neighborhoods of zero consisting of balanced and pseudoconvex sets, that is, of sets $U$ for which $\mu U \subset U$, whenever $|\mu| \leq 1$ and $U+U \subset \lambda U$ for some $\lambda \geq 2$. The topology of a locally pseudoconvex algebra can be defined by a family

Copyright 2016 by the Tusi Mathematical Research Group.

Received Nov. 3, 2015; Accepted Jan. 26, 2016.

${ }^{*}$ Corresponding author.

2010 Mathematics Subject Classification. Primary 46H05; Secondary 46H20.

Keywords. topological algebra, locally $m$-convex algebra, dense finitely generated ideals, theorem of Arens. 


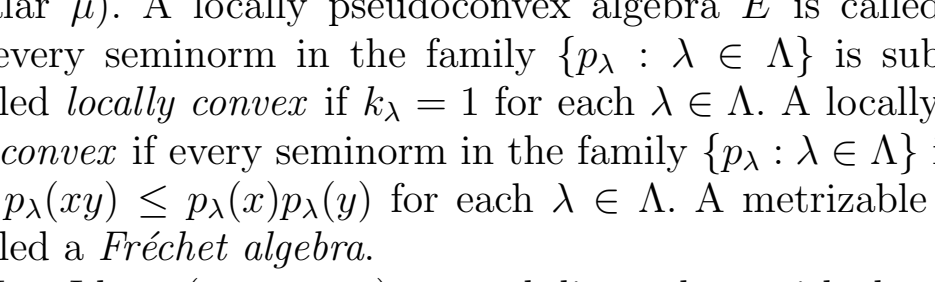

\title{
ABOUT LOCALLY $m$-CONVEX ALGEBRAS WITH DENSE FINITELY GENERATED IDEALS
}

\author{
HUGO ARIZMENDI PEIMBERT ${ }^{1}$ and REYNA MARÍA PÉREZ-TISCAREÑO ${ }^{*}$ \\ Communicated by Z. Lykova
}

\begin{abstract}
It is well known, as a consequence of a theorem of Richard Arens, that a commutative Fréchet locally $m$-convex algebra $E$ with unit does not have dense finitely generated ideals. We shall see that this result can no longer be true if $E$ is not complete and metrizable. We observe that the same is true for the theorem of Arens; that is, this theorem can no longer be true if $E$ is not complete and metrizable. Moreover, several conditions for a unital commutative (not necessarily complete) locally $m$-convex algebra are given, for which all maximal ideals have codimension one.
\end{abstract}

\section{Preliminaries}

Let $E$ be a unital topological algebra over $\mathbb{C}$ (the field of complex numbers) with separately continuous multiplication (in short, a topological algebra).

Let $B$ be a complex commutative unital algebra. We denote by $\mathfrak{M}^{\#}(B)$ the set of all nonzero multiplicative linear functionals on $B$ provided with the weakstar topology $w^{*}$. When $B$ is a topological algebra, $\mathfrak{M}(B)$ denotes the topological subspace of $\mathfrak{M}^{\#}(B)$ consisting of all nonzero multiplicative continuous linear functionals on $B$ provided with the weak-star topology $w^{*}$.

A locally pseudoconvex algebra is a topological algebra with a base of neighborhoods of zero consisting of balanced and pseudoconvex sets, that is, of sets $U$ for which $\mu U \subset U$, whenever $|\mu| \leq 1$ and $U+U \subset \lambda U$ for some $\lambda \geq 2$. The topology of a locally pseudoconvex algebra can be defined by a family

Copyright 2016 by the Tusi Mathematical Research Group.

Received Nov. 3, 2015; Accepted Jan. 26, 2016.

${ }^{*}$ Corresponding author.

2010 Mathematics Subject Classification. Primary 46H05; Secondary 46H20.

Keywords. topological algebra, locally $m$-convex algebra, dense finitely generated ideals, theorem of Arens. 


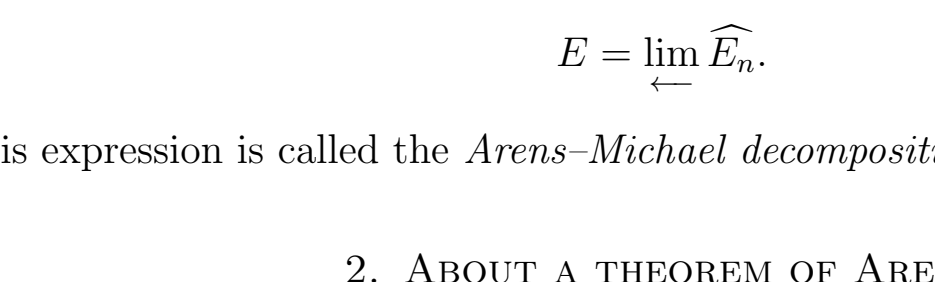

\title{
ABOUT LOCALLY $m$-CONVEX ALGEBRAS WITH DENSE FINITELY GENERATED IDEALS
}

\author{
HUGO ARIZMENDI PEIMBERT ${ }^{1}$ and REYNA MARÍA PÉREZ-TISCAREÑO ${ }^{*}$ \\ Communicated by Z. Lykova
}

\begin{abstract}
It is well known, as a consequence of a theorem of Richard Arens, that a commutative Fréchet locally $m$-convex algebra $E$ with unit does not have dense finitely generated ideals. We shall see that this result can no longer be true if $E$ is not complete and metrizable. We observe that the same is true for the theorem of Arens; that is, this theorem can no longer be true if $E$ is not complete and metrizable. Moreover, several conditions for a unital commutative (not necessarily complete) locally $m$-convex algebra are given, for which all maximal ideals have codimension one.
\end{abstract}

\section{Preliminaries}

Let $E$ be a unital topological algebra over $\mathbb{C}$ (the field of complex numbers) with separately continuous multiplication (in short, a topological algebra).

Let $B$ be a complex commutative unital algebra. We denote by $\mathfrak{M}^{\#}(B)$ the set of all nonzero multiplicative linear functionals on $B$ provided with the weakstar topology $w^{*}$. When $B$ is a topological algebra, $\mathfrak{M}(B)$ denotes the topological subspace of $\mathfrak{M}^{\#}(B)$ consisting of all nonzero multiplicative continuous linear functionals on $B$ provided with the weak-star topology $w^{*}$.

A locally pseudoconvex algebra is a topological algebra with a base of neighborhoods of zero consisting of balanced and pseudoconvex sets, that is, of sets $U$ for which $\mu U \subset U$, whenever $|\mu| \leq 1$ and $U+U \subset \lambda U$ for some $\lambda \geq 2$. The topology of a locally pseudoconvex algebra can be defined by a family

Copyright 2016 by the Tusi Mathematical Research Group.

Received Nov. 3, 2015; Accepted Jan. 26, 2016.

${ }^{*}$ Corresponding author.

2010 Mathematics Subject Classification. Primary 46H05; Secondary 46H20.

Keywords. topological algebra, locally $m$-convex algebra, dense finitely generated ideals, theorem of Arens. 


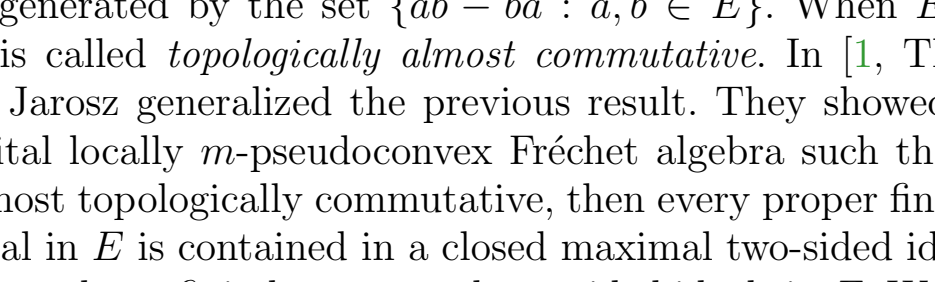

\title{
ABOUT LOCALLY $m$-CONVEX ALGEBRAS WITH DENSE FINITELY GENERATED IDEALS
}

\author{
HUGO ARIZMENDI PEIMBERT ${ }^{1}$ and REYNA MARÍA PÉREZ-TISCAREÑO ${ }^{*}$ \\ Communicated by Z. Lykova
}

\begin{abstract}
It is well known, as a consequence of a theorem of Richard Arens, that a commutative Fréchet locally $m$-convex algebra $E$ with unit does not have dense finitely generated ideals. We shall see that this result can no longer be true if $E$ is not complete and metrizable. We observe that the same is true for the theorem of Arens; that is, this theorem can no longer be true if $E$ is not complete and metrizable. Moreover, several conditions for a unital commutative (not necessarily complete) locally $m$-convex algebra are given, for which all maximal ideals have codimension one.
\end{abstract}

\section{Preliminaries}

Let $E$ be a unital topological algebra over $\mathbb{C}$ (the field of complex numbers) with separately continuous multiplication (in short, a topological algebra).

Let $B$ be a complex commutative unital algebra. We denote by $\mathfrak{M}^{\#}(B)$ the set of all nonzero multiplicative linear functionals on $B$ provided with the weakstar topology $w^{*}$. When $B$ is a topological algebra, $\mathfrak{M}(B)$ denotes the topological subspace of $\mathfrak{M}^{\#}(B)$ consisting of all nonzero multiplicative continuous linear functionals on $B$ provided with the weak-star topology $w^{*}$.

A locally pseudoconvex algebra is a topological algebra with a base of neighborhoods of zero consisting of balanced and pseudoconvex sets, that is, of sets $U$ for which $\mu U \subset U$, whenever $|\mu| \leq 1$ and $U+U \subset \lambda U$ for some $\lambda \geq 2$. The topology of a locally pseudoconvex algebra can be defined by a family

Copyright 2016 by the Tusi Mathematical Research Group.

Received Nov. 3, 2015; Accepted Jan. 26, 2016.

${ }^{*}$ Corresponding author.

2010 Mathematics Subject Classification. Primary 46H05; Secondary 46H20.

Keywords. topological algebra, locally $m$-convex algebra, dense finitely generated ideals, theorem of Arens. 


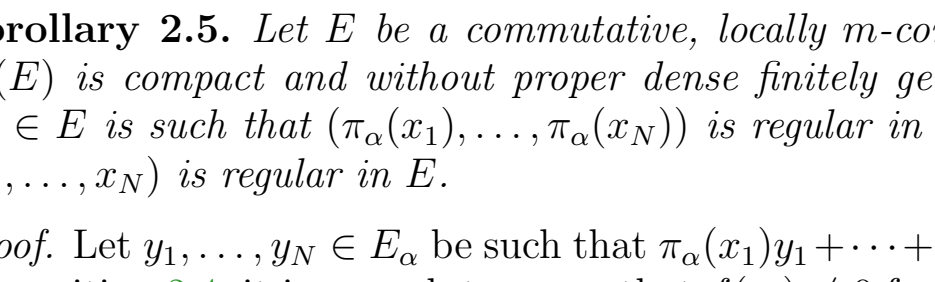

\title{
ABOUT LOCALLY $m$-CONVEX ALGEBRAS WITH DENSE FINITELY GENERATED IDEALS
}

\author{
HUGO ARIZMENDI PEIMBERT ${ }^{1}$ and REYNA MARÍA PÉREZ-TISCAREÑO ${ }^{*}$ \\ Communicated by Z. Lykova
}

\begin{abstract}
It is well known, as a consequence of a theorem of Richard Arens, that a commutative Fréchet locally $m$-convex algebra $E$ with unit does not have dense finitely generated ideals. We shall see that this result can no longer be true if $E$ is not complete and metrizable. We observe that the same is true for the theorem of Arens; that is, this theorem can no longer be true if $E$ is not complete and metrizable. Moreover, several conditions for a unital commutative (not necessarily complete) locally $m$-convex algebra are given, for which all maximal ideals have codimension one.
\end{abstract}

\section{Preliminaries}

Let $E$ be a unital topological algebra over $\mathbb{C}$ (the field of complex numbers) with separately continuous multiplication (in short, a topological algebra).

Let $B$ be a complex commutative unital algebra. We denote by $\mathfrak{M}^{\#}(B)$ the set of all nonzero multiplicative linear functionals on $B$ provided with the weakstar topology $w^{*}$. When $B$ is a topological algebra, $\mathfrak{M}(B)$ denotes the topological subspace of $\mathfrak{M}^{\#}(B)$ consisting of all nonzero multiplicative continuous linear functionals on $B$ provided with the weak-star topology $w^{*}$.

A locally pseudoconvex algebra is a topological algebra with a base of neighborhoods of zero consisting of balanced and pseudoconvex sets, that is, of sets $U$ for which $\mu U \subset U$, whenever $|\mu| \leq 1$ and $U+U \subset \lambda U$ for some $\lambda \geq 2$. The topology of a locally pseudoconvex algebra can be defined by a family

Copyright 2016 by the Tusi Mathematical Research Group.

Received Nov. 3, 2015; Accepted Jan. 26, 2016.

${ }^{*}$ Corresponding author.

2010 Mathematics Subject Classification. Primary 46H05; Secondary 46H20.

Keywords. topological algebra, locally $m$-convex algebra, dense finitely generated ideals, theorem of Arens. 


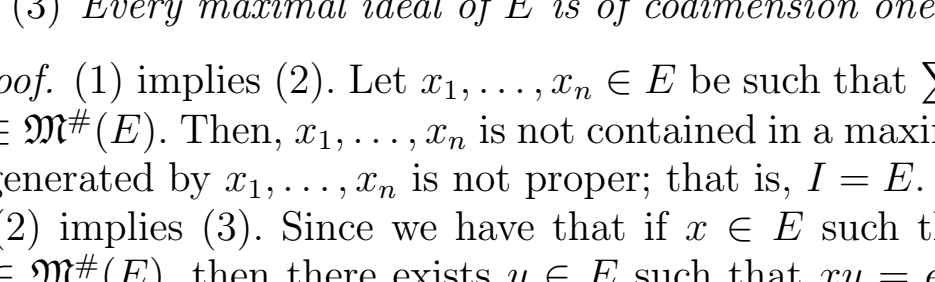

\title{
ABOUT LOCALLY $m$-CONVEX ALGEBRAS WITH DENSE FINITELY GENERATED IDEALS
}

\author{
HUGO ARIZMENDI PEIMBERT ${ }^{1}$ and REYNA MARÍA PÉREZ-TISCAREÑO ${ }^{*}$ \\ Communicated by Z. Lykova
}

\begin{abstract}
It is well known, as a consequence of a theorem of Richard Arens, that a commutative Fréchet locally $m$-convex algebra $E$ with unit does not have dense finitely generated ideals. We shall see that this result can no longer be true if $E$ is not complete and metrizable. We observe that the same is true for the theorem of Arens; that is, this theorem can no longer be true if $E$ is not complete and metrizable. Moreover, several conditions for a unital commutative (not necessarily complete) locally $m$-convex algebra are given, for which all maximal ideals have codimension one.
\end{abstract}

\section{Preliminaries}

Let $E$ be a unital topological algebra over $\mathbb{C}$ (the field of complex numbers) with separately continuous multiplication (in short, a topological algebra).

Let $B$ be a complex commutative unital algebra. We denote by $\mathfrak{M}^{\#}(B)$ the set of all nonzero multiplicative linear functionals on $B$ provided with the weakstar topology $w^{*}$. When $B$ is a topological algebra, $\mathfrak{M}(B)$ denotes the topological subspace of $\mathfrak{M}^{\#}(B)$ consisting of all nonzero multiplicative continuous linear functionals on $B$ provided with the weak-star topology $w^{*}$.

A locally pseudoconvex algebra is a topological algebra with a base of neighborhoods of zero consisting of balanced and pseudoconvex sets, that is, of sets $U$ for which $\mu U \subset U$, whenever $|\mu| \leq 1$ and $U+U \subset \lambda U$ for some $\lambda \geq 2$. The topology of a locally pseudoconvex algebra can be defined by a family

Copyright 2016 by the Tusi Mathematical Research Group.

Received Nov. 3, 2015; Accepted Jan. 26, 2016.

${ }^{*}$ Corresponding author.

2010 Mathematics Subject Classification. Primary 46H05; Secondary 46H20.

Keywords. topological algebra, locally $m$-convex algebra, dense finitely generated ideals, theorem of Arens. 


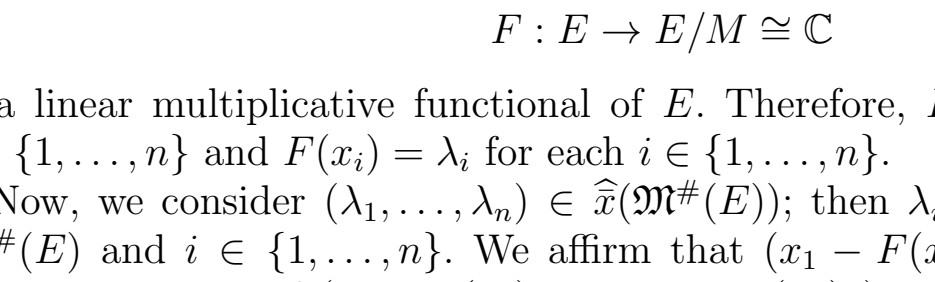

\title{
ABOUT LOCALLY $m$-CONVEX ALGEBRAS WITH DENSE FINITELY GENERATED IDEALS
}

\author{
HUGO ARIZMENDI PEIMBERT ${ }^{1}$ and REYNA MARÍA PÉREZ-TISCAREÑO ${ }^{*}$ \\ Communicated by Z. Lykova
}

\begin{abstract}
It is well known, as a consequence of a theorem of Richard Arens, that a commutative Fréchet locally $m$-convex algebra $E$ with unit does not have dense finitely generated ideals. We shall see that this result can no longer be true if $E$ is not complete and metrizable. We observe that the same is true for the theorem of Arens; that is, this theorem can no longer be true if $E$ is not complete and metrizable. Moreover, several conditions for a unital commutative (not necessarily complete) locally $m$-convex algebra are given, for which all maximal ideals have codimension one.
\end{abstract}

\section{Preliminaries}

Let $E$ be a unital topological algebra over $\mathbb{C}$ (the field of complex numbers) with separately continuous multiplication (in short, a topological algebra).

Let $B$ be a complex commutative unital algebra. We denote by $\mathfrak{M}^{\#}(B)$ the set of all nonzero multiplicative linear functionals on $B$ provided with the weakstar topology $w^{*}$. When $B$ is a topological algebra, $\mathfrak{M}(B)$ denotes the topological subspace of $\mathfrak{M}^{\#}(B)$ consisting of all nonzero multiplicative continuous linear functionals on $B$ provided with the weak-star topology $w^{*}$.

A locally pseudoconvex algebra is a topological algebra with a base of neighborhoods of zero consisting of balanced and pseudoconvex sets, that is, of sets $U$ for which $\mu U \subset U$, whenever $|\mu| \leq 1$ and $U+U \subset \lambda U$ for some $\lambda \geq 2$. The topology of a locally pseudoconvex algebra can be defined by a family

Copyright 2016 by the Tusi Mathematical Research Group.

Received Nov. 3, 2015; Accepted Jan. 26, 2016.

${ }^{*}$ Corresponding author.

2010 Mathematics Subject Classification. Primary 46H05; Secondary 46H20.

Keywords. topological algebra, locally $m$-convex algebra, dense finitely generated ideals, theorem of Arens. 


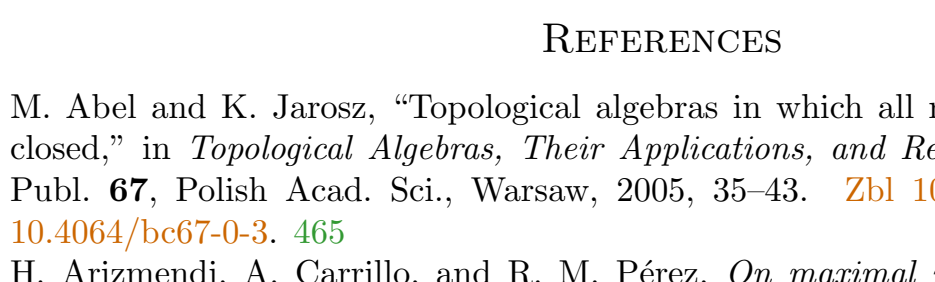

\title{
ABOUT LOCALLY $m$-CONVEX ALGEBRAS WITH DENSE FINITELY GENERATED IDEALS
}

\author{
HUGO ARIZMENDI PEIMBERT ${ }^{1}$ and REYNA MARÍA PÉREZ-TISCAREÑO ${ }^{*}$ \\ Communicated by Z. Lykova
}

\begin{abstract}
It is well known, as a consequence of a theorem of Richard Arens, that a commutative Fréchet locally $m$-convex algebra $E$ with unit does not have dense finitely generated ideals. We shall see that this result can no longer be true if $E$ is not complete and metrizable. We observe that the same is true for the theorem of Arens; that is, this theorem can no longer be true if $E$ is not complete and metrizable. Moreover, several conditions for a unital commutative (not necessarily complete) locally $m$-convex algebra are given, for which all maximal ideals have codimension one.
\end{abstract}

\section{Preliminaries}

Let $E$ be a unital topological algebra over $\mathbb{C}$ (the field of complex numbers) with separately continuous multiplication (in short, a topological algebra).

Let $B$ be a complex commutative unital algebra. We denote by $\mathfrak{M}^{\#}(B)$ the set of all nonzero multiplicative linear functionals on $B$ provided with the weakstar topology $w^{*}$. When $B$ is a topological algebra, $\mathfrak{M}(B)$ denotes the topological subspace of $\mathfrak{M}^{\#}(B)$ consisting of all nonzero multiplicative continuous linear functionals on $B$ provided with the weak-star topology $w^{*}$.

A locally pseudoconvex algebra is a topological algebra with a base of neighborhoods of zero consisting of balanced and pseudoconvex sets, that is, of sets $U$ for which $\mu U \subset U$, whenever $|\mu| \leq 1$ and $U+U \subset \lambda U$ for some $\lambda \geq 2$. The topology of a locally pseudoconvex algebra can be defined by a family

Copyright 2016 by the Tusi Mathematical Research Group.

Received Nov. 3, 2015; Accepted Jan. 26, 2016.

${ }^{*}$ Corresponding author.

2010 Mathematics Subject Classification. Primary 46H05; Secondary 46H20.

Keywords. topological algebra, locally $m$-convex algebra, dense finitely generated ideals, theorem of Arens. 